\title{
Fermi surface and nested magnetic breakdown in $\mathrm{WTe}_{2}$
}

\author{
J. F. Linnartz, ${ }^{1,2}$ C. S. A. Müller,,${ }^{1,2}$ Yu-Te Hsu, ${ }^{1,2}$ C. Breth Nielsen $\odot,{ }^{3}$ M. Bremholm, ${ }^{3}$ \\ N. E. Hussey $\odot,{ }^{1,2,4}$ A. Carrington, ${ }^{4,}{ }^{*}$ and S. Wiedmann $\oplus^{1,2, \dagger}$ \\ ${ }^{1}$ High Field Magnet Laboratory (HFML-EMFL), Radboud University, Toernooiveld 7, Nijmegen 6525 ED, Netherlands \\ ${ }^{2}$ Radboud University, Institute for Molecules and Materials, Nijmegen 6525 AJ, Netherlands \\ ${ }^{3}$ Department of Chemistry and iNANO, Aarhus University, Langelandsgade 140, DK-8000 Aarhus C, Denmark \\ ${ }^{4}$ H.H. Wills Physics Laboratory, University of Bristol, Tyndall Avenue, BS8 1TL, United Kingdom
}

(Received 21 July 2021; revised 29 October 2021; accepted 6 December 2021; published 18 January 2022)

\begin{abstract}
We report a detailed Shubnikov-de Haas $(\mathrm{SdH})$ study on the Weyl type-II semimetal $\mathrm{WTe}_{2}$ in magnetic fields up to $29 \mathrm{~T}$. By using the $\mathrm{SdH}$ results to guide our density functional theory calculations, we are able to accurately determine its Fermi surface by employing a moderate Hubbard $U$ term, which is an essential step in explaining the unusual electronic properties of this much studied material. In addition to the fundamental orbits, we observe magnetic breakdown, which can consistently be explained within the model of a Russian-doll nested Fermi surface of electron and hole pockets. The onset of magnetic breakdown in $\mathrm{WTe}_{2}$ is solely determined by impurity damping in contrast to magnetic breakdown scenarios in other metallic systems.
\end{abstract}

DOI: 10.1103/PhysRevResearch.4.L012005

The layered transition-metal dichalcogenide $\mathrm{WTe}_{2}$ provides a versatile platform to investigate topologically nontrivial phases [1]. Bulk $\mathrm{WTe}_{2}$ in the $T_{d}$ phase has been identified as the prototypical example of a Weyl type-II semimetal [2,3]. In the past few years, particular attention has been paid to the observation of a large, quadratic, and nonsaturating magnetoresistance (MR) up to $60 \mathrm{~T}$, a signature of a perfectly compensated semimetal [4]. Bound by van der Waals interaction, two-dimensional (2D) layers of $\mathrm{WTe}_{2}$ can also be exfoliated down to a monolayer. Through conventional gating techniques, various phases of matter such as the quantum spin Hall effect [5], superconductivity in a monolayer [6], and room-temperature vertical ferroelectricity in its bi- and trilayer form [7] have been observed.

The Fermi surface (FS) of bulk $\mathrm{WTe}_{2}$ has been investigated through angle-resolved photoemission spectroscopy (ARPES) [8-15] and quantum oscillation (QO) experiments [10,14,16-22]. With previous density functional theory (DFT) band-structure calculations, however, it has proved challenging to correctly explain the size and shape of the small Fermi pockets [4,8-10,12,13,15-19,22].

The material has two mirror symmetries and consequently, there are two groups of electron and hole pockets, see Fig. 2(d). The majority of experimental investigations conclude that the FS does indeed comprise two pairs of electronlike pockets and two pairs of holelike pockets, the latter located near the center of the Brillouin zone, sandwiched

\footnotetext{
*a.carrington@bristol.ac.uk

†steffen.wiedmann@ru.nl

Published by the American Physical Society under the terms of the Creative Commons Attribution 4.0 International license. Further distribution of this work must maintain attribution to the author(s) and the published article's title, journal citation, and DOI.
}

between the two pairs of electron pockets. Due to the lack of inversion symmetry in $\mathrm{WTe}_{2}$, the spin-orbit interaction leads to a Dresselhaus spin splitting of the bands and hence the electron and hole FS consist of nested Russian-doll pairs $[16,22]$. A question remains, however, regarding the exact location of the Fermi level and whether the hole pockets are connected over the $\Gamma$ point forming a dog-bone-shaped hole orbit $[9,19]$ or if additional electron pockets reside in the vicinity of the $\Gamma$ point [14].

One way to solve this longstanding puzzle is to exploit the phenomenon of magnetic breakdown (MB), i.e., tunneling of quasiparticles between distinct (adjacent) pockets of the FS above a threshold magnetic field. This leads to the formation of new orbits consisting of linear combinations of the fundamental frequencies of the associated pockets and thereby constraining further the topology of the FS. Indeed, previous QO studies have shown that MB might occur in $\mathrm{WTe}_{2}$ [16-18,21], though a robust identification of these orbits has not yet been done. Additionally, O'Brien et al. proposed that magnetic breakdown in $\mathrm{WTe}_{2}$ occurs between one electron and one hole pocket due to the specific cone structure of a Weyl type-II semimetal whereby electron and hole bands touch each other in $k$ space [23]. Knowing whether this scenario is actually manifested in $\mathrm{WTe}_{2}$ depends on a detailed knowledge of the Fermi surface, which is currently lacking.

In this Letter, we present high-field magnetotransport experiments on a mm-sized $\mathrm{WTe}_{2}$ sample down to ${ }^{3} \mathrm{He}$ temperatures. Our analysis of the observed Shubnikov-de Haas $(\mathrm{SdH})$ oscillations, when combined with our DFT calculations determines the precise electronic structure of $\mathrm{WTe}_{2}$ close to the Fermi level. Our subsequent analysis confirms the nested Russian-doll arrangement but suggests that MB can only occur between pockets of the same sign. Moreover, and in contrast with other metallic systems, the onset of MB is found to be solely determined by impurity damping. 

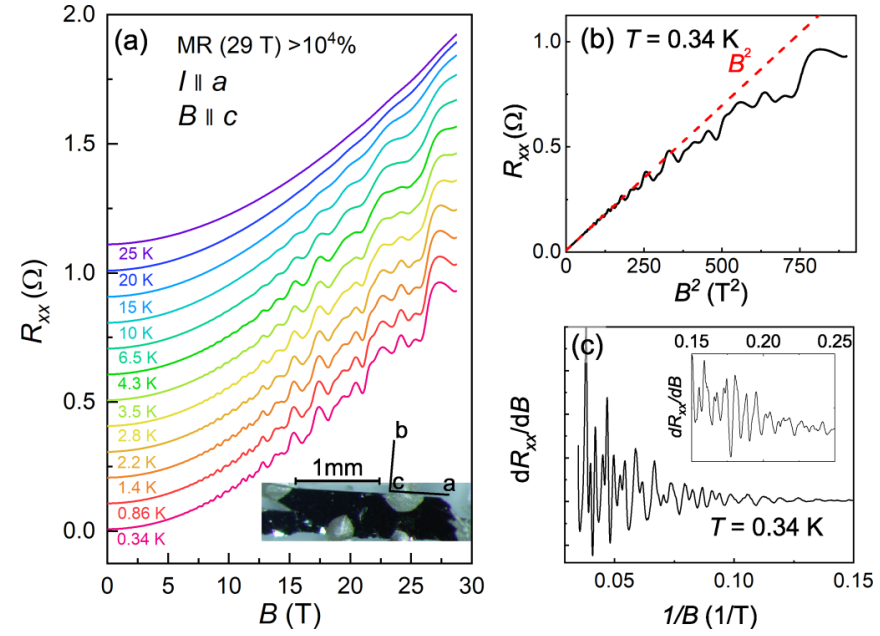

FIG. 1. (a) Resistance $R_{x x}$ as a function of magnetic field measured for various temperatures up to $25 \mathrm{~K}$. The curves are offset vertically for clarity. The inset shows the mm-sized exfoliated sample. (b) $R_{x x}$ plotted versus $B^{2}$ to highlight the quadratic behavior of the MR. (c) $d R_{x x} / d B$ as a function of $1 / B$ at $0.34 \mathrm{~K}$. The inset shows the low-field part of $d R_{x x} / d B(1 / B)$.

$\mathrm{WTe}_{2}$ flakes were grown by vapor transport method using iodine as the transport agent. Tungsten $(99.9 \%)$ and tellurium (99.9999\%) powders were ground together, pressed into a pellet and prereacted in an evacuated silica tube at $750{ }^{\circ} \mathrm{C}$ for two days. The product was then reground, pressed into a pellet again and loaded into a silica tube with iodine pieces. The tube was placed off center in a tube furnace to create a temperature gradient of $50{ }^{\circ} \mathrm{C}$ between 850 and $800^{\circ} \mathrm{C}$ and left to grow for two weeks. Figure 1(a) shows the longitudinal resistance $R_{x x}$ for the sample under study (residual resistance ratio RRR = 30) for various temperatures with the magnetic field $B$ applied along the $c$ axis while the current is passed along the $a$ axis of the sample. For all temperatures, QOs are superimposed on a $B^{2}$ MR of $\sim 12000 \%$ at maximum field. The quadratic MR persists up to $15 \mathrm{~T}$ indicating perfect charge carrier compensation before crossing over to a $B^{1.7}$ dependence up to the maximum field, see Fig. 1(b). In Fig. 1(c), $d R_{x x} / d B$ is plotted as a function of $1 / B$ in order to highlight the emergence of QOs for $B>4 \mathrm{~T}$ at $T=0.34 \mathrm{~K}$.

In Fig. 2(a), fast Fourier transforms (FFTs) of the $d R_{x x} / d B$ data of Fig. 1(c) are presented for different magnetic field ranges. For the lowest field range [4-10] T, four distinct peaks are observed, which we mark as $\alpha, \beta, \gamma$, and $\delta$ following the labeling of Ref. [16]. For details, we refer to Fig. S1 in the Supplemental Material [25]. Using the Onsager relation, we relate the frequencies $f$ observed in the FFT spectrum to the extremal areas $A_{f}$ of the individual pockets: $f=$ $(\hbar / 2 \pi e) A_{f}[26]$. Above a threshold magnetic field $(B \geqslant 8 \mathrm{~T})$, the QO pattern becomes more complex. In addition to the well-developed low-frequency peaks, a high-frequency orbit at $240 \mathrm{~T}$ appears [see the [5-28.7 T] spectrum in Fig. 2(a)]. This frequency corresponds approximately to the sum of the individual frequencies of the $\alpha$ and $\delta$ orbits, $f_{\alpha+\delta}=f_{\alpha}+f_{\delta}$ and is thus a viable signature of MB that will be discussed later.

The angle dependence of the $\mathrm{SdH}$ oscillations is presented in Fig. 2(b). $B_{\perp}$ can be determined by maximizing $R_{x x}$ due to
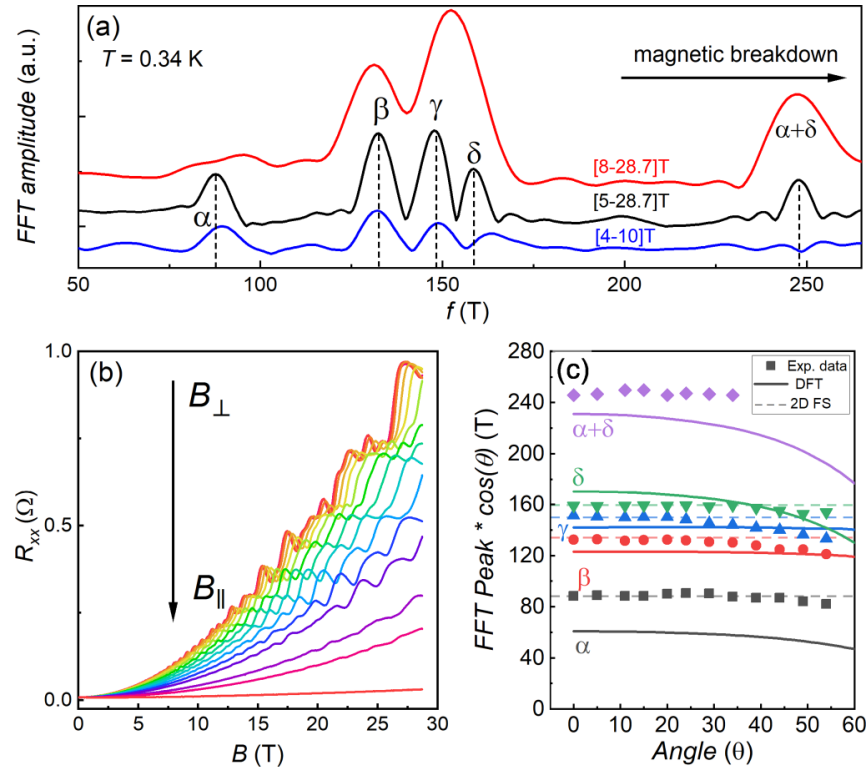

(d)

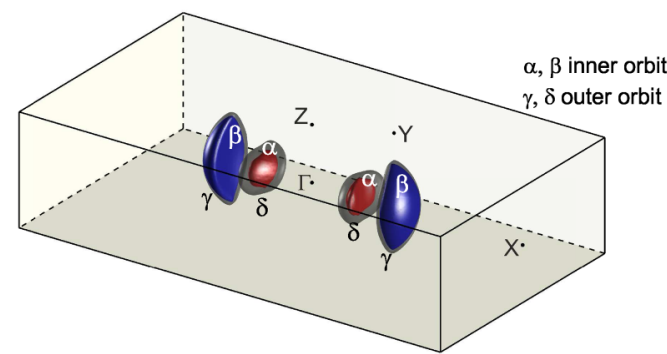

FIG. 2. (a) FFT spectrum (Hann window) of the SdH signal for various magnetic field ranges. At low $B$, the individual pockets of the FS are observed. The orbits are labeled with greek letters. With increasing field range, MB occurs and an additional orbit with frequency $f_{\alpha+\delta}$ emerges. (b) $R_{x x}$ for different tilt angles with $5^{\circ}$ increments at $T=1.4 \mathrm{~K}$. (c) Rotation from (001) to (010). Maxima of the FFT peaks as a function of tilt angle are indicated with solid symbols. The dashed lines correspond to the $1 / \cos (\theta)$ dependence expected for a two-dimensional system, the solid lines to the DFT calculation. (d) Three-dimensional FS of $\mathrm{WTe}_{2}$ obtained via LDA+U DFT calculation with $U=3 \mathrm{eV}$.

the sensitivity of the MR of $\mathrm{WTe}_{2}$ to changes in tilt angle [27]. A commercial Hall probe has been used to extract precise values for the other tilt angles. Both the MR and the QO amplitudes strongly decrease as the sample is rotated from $B_{\perp}$ (i.e., $B$ parallel to the $c$ axis) to $B_{\|}$( $B$ parallel to the $a$ axis). In order to map the $\mathrm{FS}$ of $\mathrm{WTe}_{2}$, the frequencies of peaks obtained from the FFT spectrum [5-28.7 T] multiplied by $\cos \theta$ are plotted as a function of tilt angle $\theta$ in Fig. 2(c) to better visualize the quasi-2D nature of the pockets. With increasing $\theta$, the $\mathrm{QO}$ amplitudes of the individual orbits become more and more damped and eventually vanish above $55^{\circ}$. The MB orbit, $f_{\alpha+\delta}$ can be observed up to $\theta=30^{\circ}$, as reported previously [16]. The persistence of this orbit over such a wide angular range contrasts markedly with recent observations of MB in nodal-line semimetals [28-30] where the MB orbits are found to vanish exponentially with increasing tilt angles.

To determine the Fermi surface from our extremal orbit size and mass data we use GGA+U DFT calculations [31,32] 
TABLE I. Identified orbits, extracted frequencies and cyclotron masses from the experiment in the range from [5-28.7 T] compared to the frequencies and cyclotron masses from the DFT calculations.

\begin{tabular}{lccccc}
\hline \hline Orbit & Type & $f(\mathrm{~T})$ & $m_{c}\left(m_{e}\right)$ & $f_{D F T}(\mathrm{~T})$ & $m_{c ; D F T}\left(m_{e}\right)$ \\
\hline$\alpha$ & $\mathrm{h}$ & 88 & $0.47 \pm 0.06$ & 66 & 0.43 \\
$\beta$ & $\mathrm{e}$ & 133 & $0.40 \pm 0.02$ & 134 & 0.38 \\
$\gamma$ & $\mathrm{e}$ & 148 & $0.40 \pm 0.03$ & 155 & 0.35 \\
$\delta$ & $\mathrm{h}$ & 158 & $0.46 \pm 0.04$ & 186 & 0.58 \\
\hline \hline
\end{tabular}

with an experimentally determined structure [33]. The calculated Fermi surface is shown in Fig. 2(d) and Fig. 4(e) and consists of two pairs of hole pockets and two pairs of electron pockets, which are symmetry distinct. Each pair of electron and hole pockets is nested within each other and the surfaces are symmetrically reflected in the $\Gamma-Z-Y$ plane. From this Fermi surface we identify the $\alpha$ and $\delta$ orbits as originating from the two hole pockets, and $\beta$ and $\delta$ from the two electron pockets. Each FS pocket is found to have a single extremal orbit. For such small FS volumes, small shifts in the band energies are often needed to get perfect agreement with experiment. However, for $\mathrm{WTe}_{2}$, we find that in addition a moderate $U \simeq 3 \mathrm{eV}$ must also be employed for the tungsten $d$ orbitals in order to explain the observed value of $m_{c}$ for the hole pockets. Without this, the calculated mass is a factor $\sim 2$ too high, even after shifting the band energies to match the observed frequencies. Physically, $U$ models the on-site Coulomb repulsion; an approach which has also been employed, for example, for the related material $\mathrm{MoTe}_{2}$ where a similar Hubbard $U$ value was found [34,35]. Although the agreement with experiment is not perfect, the residual differences [see Table I and Fig. 2(c)] are acceptably small. Further details of the calculations, including how the results depend on $U$, are shown in the Supplemental Material [25].

Extraction of the cyclotron masses, $m_{c}$, of the individual pockets of the FS has proven to be a challenge for the $\beta, \gamma$, and $\delta$ peaks as they are separated by only $\simeq 10 \mathrm{~T}$ in the FFT spectrum, and a proper range has to be chosen to avoid convolution of their FFT peaks. In Fig. 3(a), the range [5-28.7 T] of the FFT spectrum, taken from $d R_{x x} / d B$, is plotted for different temperatures up to $10 \mathrm{~K}$. Figures 3(b), 3(d), 3(e), 3(f) display the FFT amplitudes of the individual peaks as a function of temperature fitted to the temperature-dependent term of the Lifshitz-Kosevich formula, $R_{T}=X / \sinh [X]$ with $X=$ $\frac{2 \pi^{2} k_{B}}{\hbar e} \frac{m_{c}^{*} T}{\langle B\rangle}$, where $\langle 1 / B\rangle=\frac{\left(1 / B_{\min }\right)+\left(1 / B_{\max }\right)}{2}$. Additional details concerning the mass extraction are discussed in the Supplemental Material [25]. The experimentally extracted cyclotron masses $m_{c}$ and those obtained from DFT calculations for each individual pocket are summarized in Table I. In order to separate the FFT peaks a relatively large field range [5-28.7 T] is needed, which could in principle lead to an underestimate of the masses. However, the variation in $m_{c}$ for the $\alpha$ pocket on the field range shown in Fig. 3(c), along with additional analysis presented in the Supplemental Material [25] shows this effect is within our errors here.

A further constraint of the FS topology can be made by analyzing the MB orbits. Magnetic breakdown occurs if the applied magnetic field $B>B_{0} \approx(h / e)\left(k_{g} / 2\right)^{2}$, where $k_{g}$ is the breakdown gap in $k$ space. From our DFT calculations, we
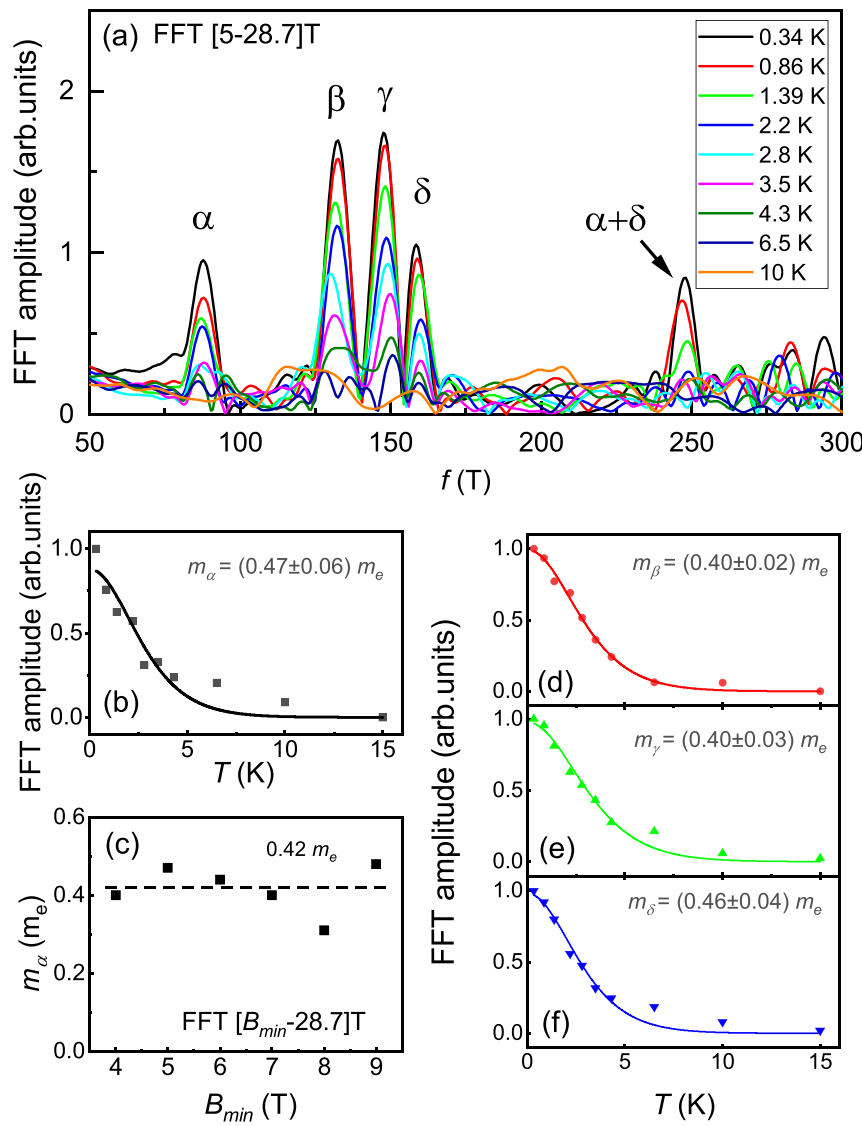

FIG. 3. (a) FFT spectra in the field range [5-28.7T] for different temperatures up to $10 \mathrm{~K}$ for $B \| c$. (b) Extraction of $m_{c}$ for the $\alpha$ pocket and (c) its corresponding range analysis in different field ranges by varying $B_{\min }$. (d)-(f) Extraction of $m_{c}$ for the other individual pockets $\beta, \gamma$, and $\delta$ of the FS.

extract a gap of $B_{0}=0.2 \mathrm{~T}$ for electrons and $0.5 \mathrm{~T}$ for holes, respectively. This implies that the Dingle field is around one order of magnitude larger than $B_{0}$, see Supplemental Material [25]. Therefore, $M B$ is determined by impurity damping in $\mathrm{WTe}_{2}$. In Fig. 4(a), the FFT spectra obtained from $d R_{x x} / d B$ containing all of the observed MR orbits are shown for several temperatures between 0.34 and $6.5 \mathrm{~K}$ in the field range [8$28.7 \mathrm{~T}]$. In addition to the $\alpha+\delta$ orbit, we identify four more MB orbits: $\beta+\gamma, 2 \alpha+\delta, \alpha+2 \delta, 3 \alpha+\delta$. Given our experimental resolution, we are only able to extract the cyclotron masses of the $\alpha+\delta, \beta+\gamma$, and $2 \alpha+\delta$ orbits, each of which corresponds to the sum of the masses of the individual pockets $[24,26,28-30]$. Their absolute values are summarized in Table II and the corresponding fits are presented in Figs. 4(b)4(d) in which the $R_{T}$ term has been fitted to the $T$-dependent FFT amplitudes.

The appropriateness of the MB scenario in $\mathrm{WTe}_{2}$ including the extracted cyclotron masses of the breakdown orbits and the persistence of the $\alpha+\delta$ orbit to large tilt angles, is corroborated by our DFT calculations. Knowing the topology of the Fermi surface and having determined the masses of the breakdown orbits enables us now to elucidate the origin of $\mathrm{MB}$ in $\mathrm{WTe}_{2}$ and thus the precise FS. The scenarios based on electron-hole MB [23] and the possibility of hole-hole 

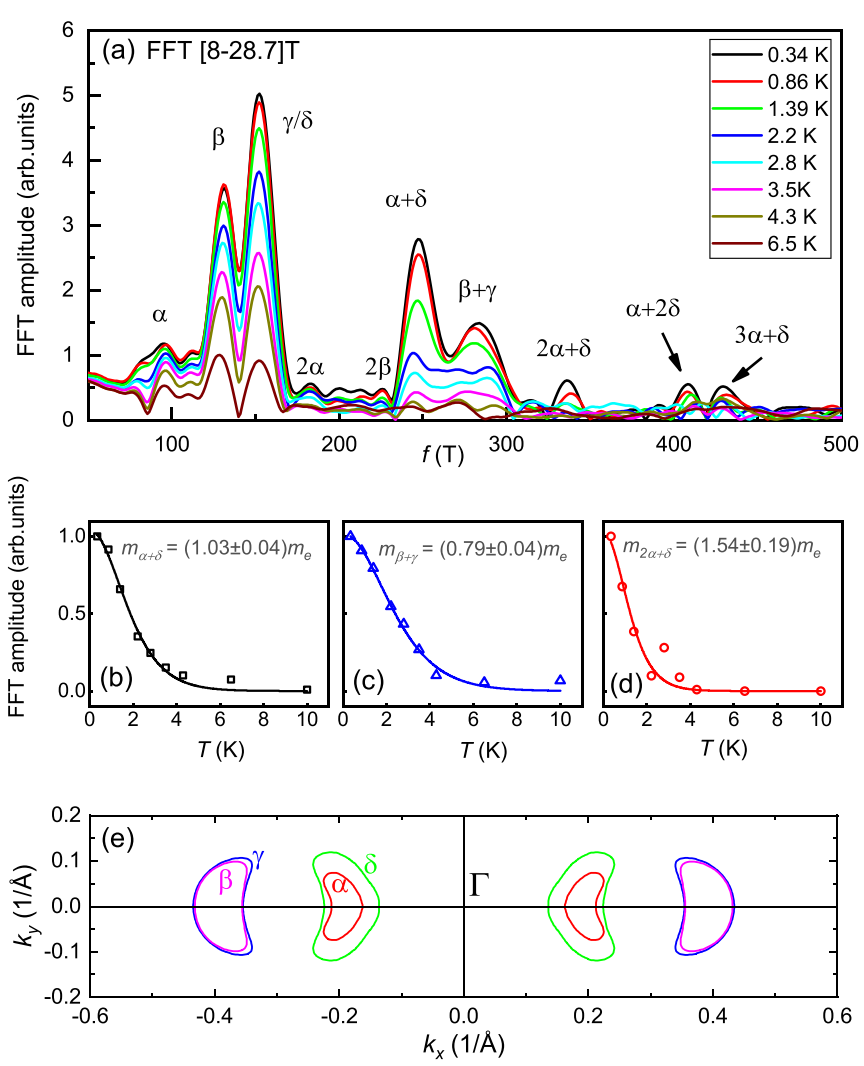

FIG. 4. (a) FFT spectrum for the field range [8-28.7 T] for different temperatures up to $6.5 \mathrm{~K}$. The corresponding individual and breakdown orbits are labeled. (b)-(d) FFT amplitude as a function of $T$ to extract $m_{c}$ of the MB orbits with the frequencies $f_{\alpha+\delta}, f_{\beta+\gamma}$ and $f_{2 \alpha+\delta}$. (e) Cut of the FS in the $k_{x}-k_{y}$ plane highlighting the nested Russian-doll configuration.

tunneling across the $\Gamma$ point [16] can be excluded due to the observed breakdown frequencies and (large) finite gaps in momentum space, see Fig. 4(e), making quasiparticle tunneling within our magnetic field range impossible. Another key observation is the absence of the $2 \delta$ pocket in our FFT spectra. If tunneling occurred over the $\Gamma$ point, QOs with a frequency $f_{2 \delta}$ would be easily observed in the FFT spectra.

In light of these considerations, the most likely scenario that is consistent with all our experimental findings is the one in which magnetic breakdown occurs between Russiandoll nested electron or hole pockets in $\mathrm{WTe}_{2}$. This scenario, supported by our DFT calculations allows for either electron-
TABLE II. Magnetic breakdown orbits and their extracted cyclotron masses using the field fitting range [8-28.7 T].

\begin{tabular}{lccc}
\hline \hline Orbit & $f(\mathrm{~T})$ & $m_{c}\left(m_{e}\right)$ & type \\
\hline$\alpha+\delta$ & 247 & $1.03 \pm 0.04$ & hole-hole \\
$\beta+\gamma$ & 283 & $0.79 \pm 0.04$ & electron-electron \\
$2 \alpha+\delta$ & 336 & $1.54 \pm 0.19$ & hole-hole \\
\hline \hline
\end{tabular}

electron $(\beta+\gamma)$ or hole-hole $(\alpha+\delta)$ tunneling, see Fig. S7 [25], within the nested pockets giving rise to frequencies of the MB orbits that we observe in the FFT spectra. The $\alpha$ pocket is situated inside the $\delta$ pocket with a similar curvature along one direction $k$ space [see Fig. 4(e)] enabling quasiparticles undergo magnetic breakdown. This scenario explains why this orbit survives out to large tilt angles as the tunneling gap will not markedly change with increasing $\theta$.

In conclusion, we have performed magnetotransport experiments up to $29 \mathrm{~T}$ combined with DFT calculations that enable us to precisely determine the Fermi surface of $\mathrm{WTe}_{2}$. Four individual pockets and their corresponding cyclotron masses have been identified and extracted. The Fermi surface exhibits quasi-2D behavior upon tilting the magnetic field away from the $c$ axis of the crystal. All observed orbits originating from magnetic breakdown have been assigned that further constrains the topology of the Fermi surface. The unprecedented resolution of our high-field study enables us to extract the cyclotron masses of MB orbits and together with the longevity of one of the MB orbits at finite tilt angles, allows for an unambiguous determination of magnetic breakdown in $\mathrm{WTe}_{2}$ that is seen to occur between two electron and hole pockets in a nested Russian-doll configuration.

This work was supported by HFML-RU/NWO-I, a member of the European Magnetic Field Laboratory (EMFL) and by the UK Engineering and Physical Sciences Research Council (Grant No. EP/R011141/1). This publication is part of the project TOPCORE (OCENW.GROOT.2019.048) of the research program NWO - GROOT which is financed by the Dutch Research Council (NWO). We gratefully acknowledge funding from the VILLUM FOUNDATION via the Centre of Excellence for Dirac Materials (11744). M.B. acknowledges the Danish Council for Independent Research, Natural Sciences under the Sapere Aude program (Grant No. 702700077B). We thank Malte Rösner and Kamran Behnia for helpful discussions.
[1] X.-C. Pan, X. Wang, F. Song, and B. Wang, The study on quantum material $\mathrm{WTe}_{2}$, Adv. Phys. X 3, 1468279 (2018).

[2] A. Soluyanov, D. Gresch, Z. Wang, Q. S. Wu, M. Troyer, X. Dai, and B. A. Bernevig, Type-II Weyl semimetals, Nature (London) 527, 495 (2015).

[3] N. P. Armitage, E. J. Mele, and A. Vishwanath, Weyl and Dirac semimetals in three-dimensional solids, Rev. Mod. Phys. 90, 015001 (2018).

[4] M. Ali, J. Xiong, S. Flynn, J. Tao, Q. D. Gibson, L. M. Schoop, T. Liang, N. Haldolaarachchige, M. Hirschberger, N. P. Ong, and R. J. Cava, Large, non-saturating magnetoresistance in $\mathrm{WTe}_{2}$, Nature (London) 514, 205 (2014).
[5] S. Wu, V. Fatemi, Q. D. Gibson, K. Watanabe, T. Taniguchi, R. J. Cava, and P. Jarillo-Herrero, Observation of the quantum spin Hall effect up to 100 Kelvin in a monolayer crystal, Science 359, 76 (2018).

[6] E. Sajadi, T. Palomaki, Z. Fei, W. Zhao, P. Bement, C. Olsen, S. A. Luescher, X. Xu, J. A. Folk, and D. H. Cobden, Gateinduced superconductivity in a monolayer topological insulator, Science 362, 922 (2018).

[7] Z. Fei, W. Zhao, T. A. Palomaki, B. Sun, M. K. Miller, Z. Zhao, J. Yan, X. Xu, and D. H. Cobden, Ferroelectric switching of a two-dimensional metal, Nature (London) 560, 336 (2018). 
[8] I. Pletikosić, M. N. Ali, A. V. Fedorov, R. J. Cava, and T. Valla, Electronic Structure Basis for the Extraordinary Magnetoresistance in $\mathrm{WTe}_{2}$, Phys. Rev. Lett. 113, 216601 (2014).

[9] J. Jiang, F. Tang, X. C. Pan, H. M. Liu, X. H. Niu, Y. X. Wang, D. F. Xu, H. F. Yang, B. P. Xie, F. Q. Song, P. Dudin, T. K. Kim, M. Hoesch, P. K. Das, I. Vobornik, X. G. Wan, and D. L. Feng, Signature of Strong Spin-Orbital Coupling in the Large Nonsaturating Magnetoresistance Material $\mathrm{WTe}_{2}$, Phys. Rev. Lett. 115, 166601 (2015).

[10] Y. Wu, N. H. Jo, M. Ochi, L. Huang, D. Mou, S. L. Bud'ko, P. C. Canfield, N. Trivedi, R. Arita, and A. Kaminski, TemperatureInduced Lifshitz Transition in $\mathrm{WTe}_{2}$, Phys. Rev. Lett. 115, 166602 (2015).

[11] Y. Wu, D. Mou, N. H. Jo, K. Sun, L. Huang, S. L. Bud'ko, P. C. Canfield, and A. Kaminski, Observation of Fermi arcs in the type-II Weyl semimetal candidate $\mathrm{WTe}_{2}$, Phys. Rev. B 94, 121113(R) (2016).

[12] C. Wang, Y. Zhang, J. Huang, S. Nie, G. Liu, A. Liang, Y. Zhang, B. Shen, J. Liu, C. Hu, Y. Ding, D. Liu, Y. Hu, S. He, L. Zhao, L. Yu, J. Hu, J. Wei, Z. Mao, Y. Shi et al., Observation of Fermi arc and its connection with bulk states in the candidate type-II Weyl semimetal $\mathrm{WTe}_{2}$, Phys. Rev. B 94, 241119(R) (2016).

[13] D. Di Sante, P. K. Das, C. Bigi, Z. Ergönenc, N. Gürtler, J. A. Krieger, T. Schmitt, M. N. Ali, G. Rossi, R. Thomale, C. Franchini, S. Picozzi, J. Fujii, V. N. Strocov, G. Sangiovanni, I. Vobornik, R. J. Cava, and G. Panaccione, Three-Dimensional Electronic Structure of the Type-II Weyl Semimetal $\mathrm{WTe}_{2}$, Phys. Rev. Lett. 119, 026403 (2017).

[14] Y. Wu, N. H. Jo, D. Mou, L. Huang, S. L. Bud'ko, P. C. Canfield, and A. Kaminski, Three-dimensionality of the bulk electronic structure in $\mathrm{WTe}_{2}$, Phys. Rev. B 95, 195138 (2017).

[15] P. K. Das, D. Di Sante, F. Cilento, C. Bigi, D. Kopic, D. Soranzio, A. Sterzi, J. A. Krieger, I. Vobornik, J. Fujii, T. Okuda, V. N. Strocov, M. B. H. Breese, F. Parmigiani, G. Rossi, S. Picozzi, R. Thomale, G. Sangiovanni, R. J. Cava, and G. Panaccione, Electronic properties of candidate type-II Weyl semimetal $\mathrm{WTe}_{2}$. A review perspective, Electron. Struct. 1, 014003 (2019).

[16] Z. Zhu, X. Lin, J. Liu, B. Fauqué, Q. Tao, C. Yang, Y. Shi, and K. Behnia, Quantum Oscillations, Thermoelectric Coefficients, and the Fermi Surface of Semimetallic WTe 2 , Phys. Rev. Lett. 114, 176601 (2015).

[17] P. L. Cai, J. Hu, L. P. He, J. Pan, X. C. Hong, Z. Zhang, J. Zhang, J. Wei, Z. Q. Mao, and S. Y. Li, Drastic Pressure Effect on the Extremely Large Magnetoresistance in $\mathrm{WTe}_{2}$ : Quantum Oscillation Study, Phys. Rev. Lett. 115, 057202 (2015).

[18] D. Rhodes, S. Das, Q. R. Zhang, B. Zeng, N. R. Pradhan, N. Kikugawa, E. Manousakis, and L. Balicas, Role of spin-orbit coupling and evolution of the electronic structure of WTe2 under an external magnetic field, Phys. Rev. B 92, 125152 (2015).

[19] X. C. Pan, Xu. Chen, H. Liu, Y. Feng, Z. Wei, Y. Zhou, Z. Chi, L. Pi, F. Yen, F. Song, X. Wan, Z. Yang, B. Wang, G. Wang, and Y. Zhang, Pressure-driven dome-shaped superconductivity and electronic structural evolution in tungsten ditelluride, Nat. Commun. 6, 7805 (2015).

[20] K. G. Rana, F. K. Dejene, N. Kumar, C. R. Rajamathi, K. Sklarek, C. Felser, and S. S. P. Parkin, Thermopower and uncon- ventional nernst effect in the predicted Type-II weyl semimetal $\mathrm{WTe}_{2}$, Nano Lett. 18, 6591 (2018).

[21] S. Onishi, R. Jha, A. Miyake, R. Higashinaka, T. D. Matsuda, M. Tokunaga, and Y. Aoki, Deviation from the Kohler's rule and Shubnikov-de Haas oscillations in type-II Weyl semimetal $\mathrm{WTe}_{2}$ : High magnetic field study up to $56 \mathrm{~T}$, AIP Adv. 8, 101330 (2018).

[22] N. H. Jo, L.-L. Wang, P. P. Orth, S. L. Bud'ko, and P. C. Canfield, Magnetoelastoresistance in $\mathrm{WTe}_{2}$ : Exploring electronic structure and extremely large magnetoresistance under strain, Proc. Natl. Acad. Sci. USA 116, 25524 (2019).

[23] T. E. O’Brien, M. Diez, and C. W. J. Beenakker, Magnetic Breakdown and Klein Tunneling in a Type-II Weyl Semimetal, Phys. Rev. Lett. 116, 236401 (2016).

[24] M. I. Kaganov, and A. A. Slutskin, Coherent magnetic breakdown, Phys. Rep. 98, 189 (1983).

[25] See Supplemental Material at http://link.aps.org/supplemental/ 10.1103/PhysRevResearch.4.L012005 for details on the assignment of individual pockets of the Fermi surface, DFT band-structure calculations, mass determination, and magnetic breakdown in the nested Russian-doll configuration.

[26] D. Shoenberg, Magnetic Oscillations in Metals (Cambridge University Press, Cambridge, 1984).

[27] L. R. Thoutam, Y. L. Wang, Z. L. Xiao, S. Das, A. Luican-Mayer, R. Divan, G. W. Crabtree, and W. K. Kwok, Temperature-Dependent Three-Dimensional Anisotropy of the Magnetoresistance in $\mathrm{WTe}_{2}$, Phys. Rev. Lett. 115, 046602 (2015).

[28] S. Pezzini, M. R. van Delft, L. M. Schoop, B. V. Lotsch, A. Carrington, M. I. Katsnelson, N. E. Hussey, and S. Wiedmann, Unconventional mass enhancement around the Dirac nodal loop in $\mathrm{ZrSiS}$, Nat. Phys. 14, 178 (2018).

[29] M. R. van Delft, S. Pezzini, T. Khouri, C. S. A. Müller, M. Breitkreiz, L. M. Schoop, A. Carrington, N. E. Hussey, and S. Wiedmann, Electron-Hole Tunneling Revealed by Quantum Oscillations in the Nodal-Line Semimetal HfSiS, Phys. Rev. Lett. 121, 256602 (2018).

[30] C. S. A. Müller, T. Khouri, M. R. van Delft, S. Pezzini, Y.-T. Hsu, J. Ayres, M. Breitkreiz, L. M. Schoop, A. Carrington, N. E. Hussey, and S. Wiedmann, Determination of the Fermi surface and field-induced quasiparticle tunneling around the Dirac nodal loop in ZrSiS, Phys. Rev. Research 2, 023217 (2020).

[31] P. Blaha, K. Schwarz, G. K. H. Madsen, D. Kvasnicka, and J. Luitz, WIEN2k (Karlheinz Schwarz, Techn. Universität Wien, Austria, 2001).

[32] J. P. Perdew, K. Burke, and M. Ernzerhof, Generalized Gradient Approximation Made Simple, Phys. Rev. Lett. 77, 3865 (1996).

[33] A. Mar, S. Jobic, and J. A. Ibers, Metal-metal vs telluriumtellurium bonding in $\mathrm{WTe}_{2}$ and its ternary variants $\mathrm{TaIrTe}_{4}$ and $\mathrm{NbIrTe}_{4}$, J. Am. Chem. Soc. 114, 8963 (1992).

[34] N. Aryal and E. Manousakis, Imporance of electron correlations in understanding photoelectron spectroscopy and Weyl character of $\mathrm{MoTe}_{2}$, Phys. Rev. B 99, 035123 (2019).

[35] Y. J. Hu, W. C. Yu, K. To Lai, D. Sun, F. F. Balakirev, W. Zhang, J. Y. Xie, K. Y. Yip, E. I. Paredes Aulestia, R. Jha, R. Higashinaka, T. D. Matsuda, Y. Yanase, Y. Aoki, and S. K. Goh, Detection of Hole Pockets in the Candidate Type-II Weyl Semimetal $\mathrm{MoTe}_{2}$ from Shubnikov-de Haas Quantum Oscillations, Phys. Rev. Lett. 124, 076402 (2020). 